\title{
Double Square Waveguide Directional Coupler for Polarimeter Calibration
}

\author{
Beatriz Aja, Enrique Villa, Luisa de la Fuente, and Eduardo Artal, Life Member, IEEE
}

\begin{abstract}
A novel full-band square waveguide coupler design based on directional couplers which couple the $T_{10}$ and $T E_{01}$ orthogonal modes in a square waveguide is presented. This waveguide coupler is aimed at calibration of polarization receivers. This is composed of a pair of rectangular waveguide directional couplers, which are rotated $90^{\circ}$ between them and both are coupled to a main square waveguide through each one of the square section walls. The coupler covers the full frequency band from $10 \mathrm{GHz}$ to $18.9 \mathrm{GHz}$. It has inherent low cross-polarization, which allows to obtain any known elliptically polarized wave at a square waveguide when a signal is applied to the couplers. The fabricated prototype of this coupler exhibits $31 \mathrm{~dB}$ of coupling, with flatness of $\pm 3.8 \mathrm{~dB}$ and excellent cross polarization better than $50 \mathrm{~dB}$ over the whole band.
\end{abstract}

Index Terms - Waveguide coupler, microwave passive components, polarization, directional couplers, polarimetric radiometers.

\section{INTRODUCTION}

$\mathrm{D}$ IFFERENT polarimetric radiometer architectures have been developed with the aim of measuring the polarization of sky observations or geophysical purposes [1]-[10]. These polarimetric radiometers are very sensitive receiver instruments used for accurately measuring the Stokes parameters of polarized incident waves. The calibration of those instruments at microwave and millimeter waves is performed through different techniques. The use of a Dicke-switch [11], in which the reference signals are radiated from a black body at two different physical temperatures, is a common calibration technique for the entire receiver chain of radiometers. A method switching between internal noise injection and external reference loads has also been demonstrated to accurately calibrate radiometer gains and offsets [12]. But, on the other hand, wave polarization angle cannot be calibrated with those calibration techniques, although they are useful for conventional microwave radiometers measuring total brightness temperature.

In polarimetric radiometers the aim is to measure the Stokes parameters which define linearly and circularly polarized brightness temperature components. Therefore, the calibration

Manuscript received July 24, 2018, revised November 14, 2018; accepted January 19, 2019. This work was supported by the Spanish Ministry of Economy and Competitiveness under Grant ESP2015-70646-C2-2-R.

B. Aja, L. de la Fuente and E. Artal are with the Department of Communication Engineering. University of Cantabria, 39005 Santander, Spain (e-mail: ajab@unican.es). of a fully polarimetric radiometer requires test signals with different well known polarizations, which number depends on the number of Stokes parameter outputs to calibrate.

The Stokes parameters for determining polarization in terms of two linearly polarized components of the electric field in standard Cartesian basis $(\hat{x}, \hat{y})$ are

$$
Q \equiv\left\langle E_{x}^{2}\right\rangle-\left\langle E_{y}^{2}\right\rangle, U \equiv 2 \operatorname{Re}\left\langle E_{x} E_{y}^{*}\right\rangle, V \equiv-2 \operatorname{Im}\left\langle E_{x} E_{y}^{*}\right\rangle .
$$

Some experiments devoted to measure the polarization of the cosmic microwave background use polarized astronomical sources for instruments calibration [1], [13]. On the other hand, the use of manufactured calibrators for polarimetric radiometer calibration is also commonly used, with a classic method consisting of the use of a linearly polarized reference load through two absorbing microwave black bodies and a polarization-selecting wire grid between them, which generates three reference noise levels [14]. Other similar methods consist in observing rotatable dielectric sheets to generate a partially polarized signal, where a dielectric sheet acts as a beam splitter transmitting most of the sky radiation but reflecting a small polarized fraction of the radiation from an ambient load perpendicular to the beam [15], [16], or polarization selecting wire-grids mounted on a mechanical rotating ring [17]. Additionally to those methods, a phase retardation plate can be added to produce an extra reference temperature with circular polarization and calibrate fully polarimetric radiometers [18][21]. Furthermore, other calibration methods apply the injection of polarized test signals through their components, such as correlated noise calibration standard coupled with directional couplers [22], an injector of reference signals with coaxial probes inserted in a circular waveguide [23], directional couplers [3] or circular waveguide directional coupler [24], [25]. These last two works have demonstrated waveguide couplers using a circular waveguide, nevertheless those directional couplers only allow to inject in the circular waveguide a broadband signal to produce a pure linearly polarized wave with a fixed angle. On the other hand, the injector presented in [23] allows to inject differently linearly polarized reference waves in a narrow band, without directivity and achieving a low level of parasitic circularly polarized wave.

E. Villa was with the Department of Communication Engineering. University of Cantabria, 39005 Santander, Spain. He is now with the Instituto de Astrofísica de Canarias, IACTec, Via Láctea s/n, 38205 La Laguna, Spain (e-mail: evilla@iac.es). 
It consists of three probes inserted in circular waveguide in which the mechanical manufacturing involving penetration and position of the probes is crucial for obtaining the required performance. Nevertheless, that solution needs a wide diameter of the circular waveguide to better control the required coupling and fabrication tolerances, which reduces the working frequency band for being operated a multimodal regime.

With the purpose of calibrating a fully polarimetric receiver, in which at least three linearly polarized waves and one circularly polarized wave are needed, a solution based on waveguide couplers to inject test signals is proposed. For this design, waveguide aperture directional couplers, which are based on Bethe's theory about small aperture coupling [26], [27], have been designed. Aperture couplers work by the interaction of two metallic waveguides through holes in their common walls. These couplers are used to obtain high directivity over broadband frequency ranges [28]-[31]. Moreover, the technique of crossed-waveguide directional couplers is also used, which provides the compactness and simplicity for the fabrication. Besides, although a common way used to improve directivity are crossed slots as coupling apertures, rectangular slots are implemented achieving good performance.

In this work, a novel square waveguide coupler based on rectangular waveguide directional couplers is presented. This coupler allows to inject two broadband signals to the rectangular waveguides and to obtain an elliptically polarized wave in the square waveguide. Moreover, when those signals are in-phase coupled to the square waveguide, a linear polarization is obtained and its angle can be controlled with the amplitude of one of the injected signals. This would allow to calibrate three out of four Stokes parameters. In addition, an external phase shift of $90^{\circ}$ in one of the coupled signal produces a circularly polarized wave. Furthermore, this coupler avoids the use of complex mechanical rotating structures with wiregrids needed for polarization selection of incoming signals in polarimeter calibration, as well as being mechanically simple to manufacture. The detailed design and manufacturing of the proposed square waveguide directional coupler are described in Section II. Its experimental results are presented in Section III. Finally, conclusions are explained in Section IV.

\section{Square Waveguide Coupler Design}

The double square waveguide coupler is composed of two identical couplers which are connected through the square waveguide rotated $90^{\circ}$ between them as it is shown in Fig. 1. This combination of the two couplers results in a dual mode coupler which allows to provide a known elliptically polarized wave in the square waveguide when two signals are applied to each individual waveguide coupler. The physical structure of this dual mode coupler has inherent low cross-polarization, since the coupled modes into the square waveguide from each coupler are orthogonal. Moreover, this coupler with square waveguide has the advantage of being easy to fabricate with Eplane split blocks.

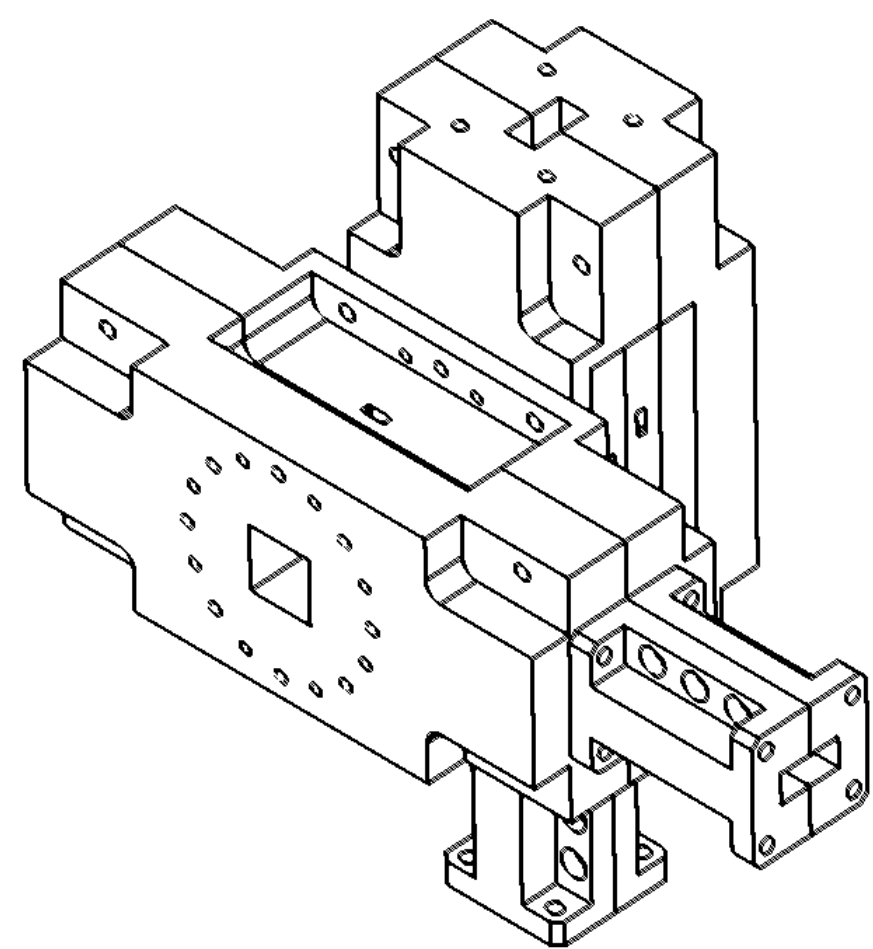

Fig. 1. General view of the dual mode coupler structure.

\section{A. Coupler Design}

The design of the coupler is based on the design of a circular waveguide directional coupler described in [24], but with the improvement of being able to produce linearly polarized wave with any angle or circular polarization, as opposed to only a linearly polarized wave with fixed angle. The goals are a coupling of around $30 \mathrm{~dB}$ in the frequency band from 10 to 18.9 GHz. This coupling is chosen for injecting a calibration signal into a receiver, taking also into account the incoming noise power level to the receiver when none calibration signal is applied and a termination is connected to the coupler.

The WR62 standard $(a=15.80 \mathrm{~mm}, b=7.90 \mathrm{~mm})$ is used for the rectangular waveguides and a square waveguide of side $16.8 \mathrm{~mm}$ is adopted in the design of the coupler.

An electromagnetic 3D model of the double directional coupler with the six physical ports is depicted in Fig. 2. Each directional coupler is composed of a waveguide power divider, which comprises two parallel waveguides coupled in their broadwall, to a crossed square waveguide in its opposite walls. The couplings of each parallel rectangular waveguides induce the components of the $\mathrm{TE}_{10}$ mode or the $\mathrm{TE}_{01}$ mode in the square waveguide, but not both, since for a mode the fields are added in phase and for the other one out of phase.

According to Fig. 2, regarding the rectangular waveguide port\#1 and port\#2 of the double directional coupler, port\#5 is the coupled port and port\#6 is the isolated one. Since the double directional coupler is symmetric, for the rectangular waveguide port\#3 and port\#4 the coupled port is port\#6, and the isolated one is port\#5. Each square waveguide port can be represented as two electrical ports, one for each propagating mode $\mathrm{TE}_{10}(\mathrm{X})$ and $\mathrm{TE}_{01}(\mathrm{Y})$. The complete S-parameters matrix of the waveguide coupler with all the electrical ports is given as: 


$\left[\begin{array}{cccccccc}S_{11} & S_{12} & S_{13} & S_{14} & C 1_{5 Y} & C 1_{5 X} & I 1_{6 Y} & I 1_{6 X} \\ S_{12} & S_{22} & S_{23} & S_{24} & C 2_{5 Y} & C 2_{5 X} & I 2_{6 Y} & I 2_{6 X} \\ S_{13} & S_{23} & S_{33} & S_{34} & I 1_{5 Y} & I 1_{5 X} & C 1_{6 Y} & C 1_{6 X} \\ S_{14} & S_{42} & S_{34} & S_{44} & I 2_{5 Y} & I 2_{5 X} & C 2_{6 Y} & C 2_{6 X} \\ C 1_{5 Y} & C 2_{5 Y} & I 1_{5 Y} & I 2_{5 Y} & S_{55 Y} & S_{55 Y X} & T_{Y} & T_{Y X} \\ C 1_{5 X} & C 2_{5 X} & I 1_{5 X} & I 2_{5 X} & S_{55 Y X} & S_{55 X} & T_{Y X} & T_{X} \\ I 1_{6 Y} & I 2_{6 Y} & C 1_{6 Y} & C 2_{6 Y} & T_{Y} & T_{Y X} & S_{66 Y} & S_{66 Y X} \\ I 1_{6 X} & I 2_{6 X} & C 1_{6 X} & C 2_{6 X} & T_{6 X 5 Y} & T_{X} & S_{66 Y X} & S_{66 X}\end{array}\right]$

where $\mathrm{C} 1$ and I1 are the coupling and isolation parameters of the Coupler\#1 and C2 and I2 are the coupling and isolation parameters of the Coupler\#2 respectively. Sii and $\mathrm{Sij}$ indicate the reflection and transmission coefficients of the rectangular ports. In the square waveguide, $T_{Y}, T_{X}$ and $T_{Y X}$ are the transmission coefficients, and $\mathrm{Sii}_{\mathrm{V}}, \mathrm{Sii}_{\mathrm{H}}, \mathrm{Sii}_{\mathrm{VH}}$ are the reflection coefficients for each propagating mode.

The coupling parameters $\mathrm{C} 1$ and $\mathrm{C} 2$ have ideally no phase difference, because the electrical length of the square waveguide section between both couplers is compensated with additional sections of rectangular waveguide, connected one to the port\#1 of Coupler\#1 and the other one to port\#4 of Coupler\#2. These sections of rectangular waveguide have a width of $16.8 \mathrm{~mm}$, like the side of the square waveguide (a_s), in order to have the same phase constant $\beta$, and a length of $51.4 \mathrm{~mm}(\mathrm{Ls} 2+\mathrm{a})$, which compensates the propagation delay between Coupler\#1 and Coupler\#2. Therefore, a known elliptically polarized wave in the square waveguide is obtained when signals with a general phase difference are applied to each waveguide coupler. Moreover, if the signal is applied to only one of the couplers, the resultant wave at the square waveguide port is a linearly polarized wave, horizontal or vertical depending on the feeding coupler. Furthermore, in-phase signals simultaneously applied to Coupler\#1 and Coupler\#2 produce an output signal coupled to the square waveguide with linear polarization in the whole frequency band, and its angle depends on the amplitudes of the applied signals.

The structure has been simulated with the FEM-based software ANSYS ${ }^{\circledR}$ HFSS Electronics Desktop using driven model. Fig. 2 shows the HFSS model of the 3D structure. Waveguide walls are taken as copper material and inside boxes are taken as vacuum.

\section{B. Prototype fabrication}

The two waveguide couplers are connected through the square waveguide with a rotation of $90^{\circ}$ between them. The double coupler is simple to fabricate with four E-plane split blocks, with two of them composing each waveguide coupler, and the two waveguide sections with $\mathrm{a}_{-} \mathrm{s}=16.8 \mathrm{~mm}$ to compensate the phase of the square waveguide between couplers.

In Fig. 3, there are two views of the two directional couplers to square waveguide, comprising four pieces all together. This view has not the sections of rectangular waveguide used to compensate the phase of the square waveguide between couplers. The view to the right shows two out of four rectangular holes of each coupler section. The dimensions indicated in Fig. 3 are summarized in Table I.

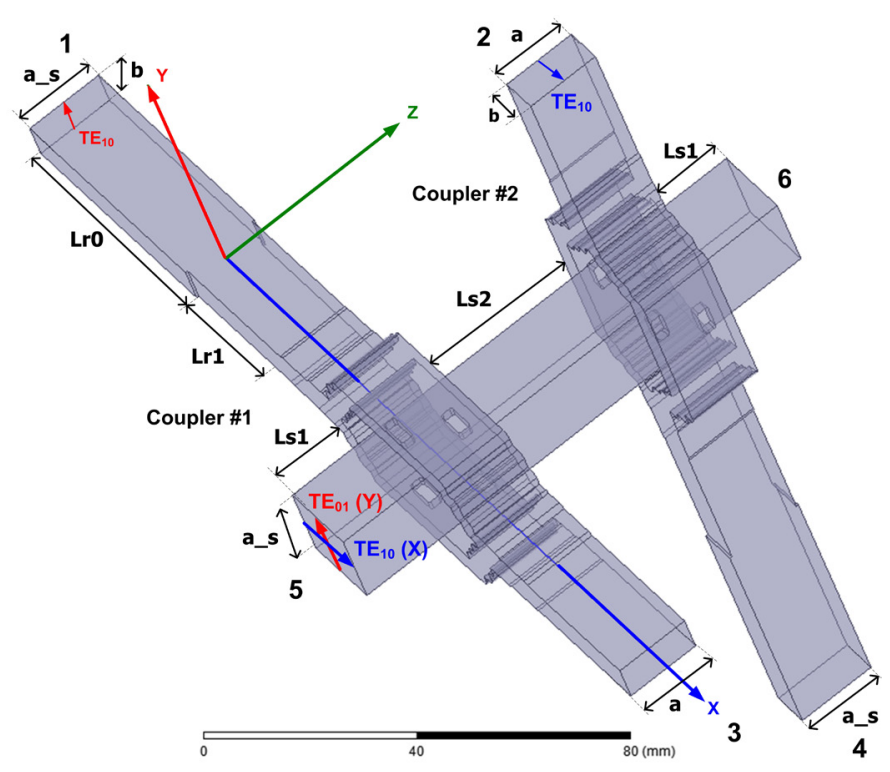

Fig. 2. Electromagnetic model of the double directional coupler to square waveguide. Port\# 5 is the coupled port and port\# 6 is the isolated one both respect to the rectangular waveguide port\#1 and port\#2. Dimensions are in Table I.

The internal machining of each block is identical, and the rectangular waveguide is split in two branches bordering the square waveguide as shown in the internal section in Fig. 4. Then, each block has two rectangular through holes from the branches of the rectangular waveguide to the square waveguide, whose shape and position are critical because they determine the performance of the coupler. All the dimensions of the coupler are summarized in Table I (see Fig. 2, Fig. 3 and Fig. 4).

An aluminum prototype is fabricated composed of the two split block waveguide parts, which are shown in Fig. 5. The complete double directional coupler to square waveguide, with waveguide sections in two of the rectangular waveguide ports to compensate phases, and waveguide matched terminations in two of the rectangular waveguide ports named 3 and 4 is shown in Fig. 6.

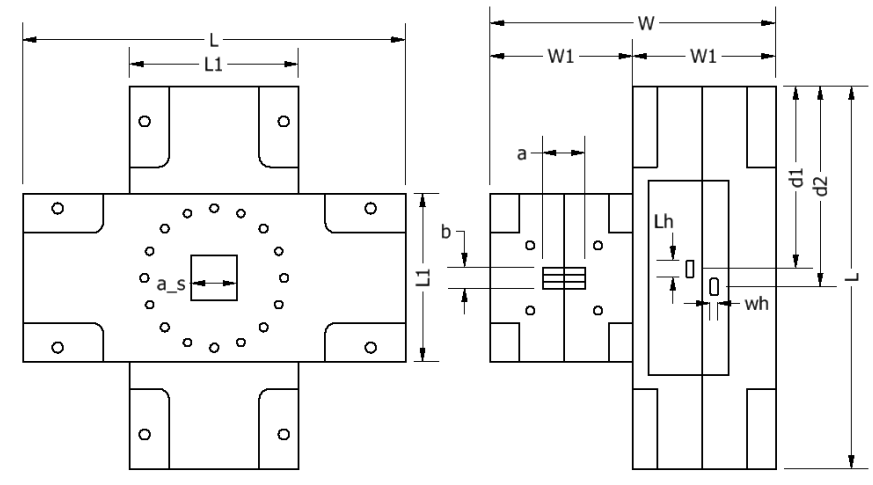

Fig. 3. External view of the four pieces comprising the two directional couplers to square waveguide, without the sections of rectangular waveguide used to compensate the phase of the square waveguide between couplers. Each coupler is composed of two pieces. Dimensions are in Table I. 


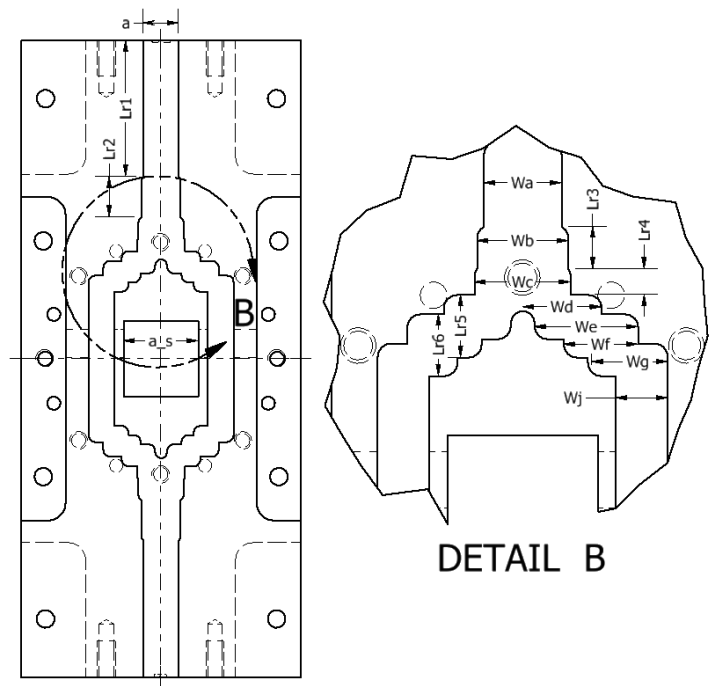

Fig. 4. Internal section of the rectangular to square waveguide coupler. Dimensions are in Table I.

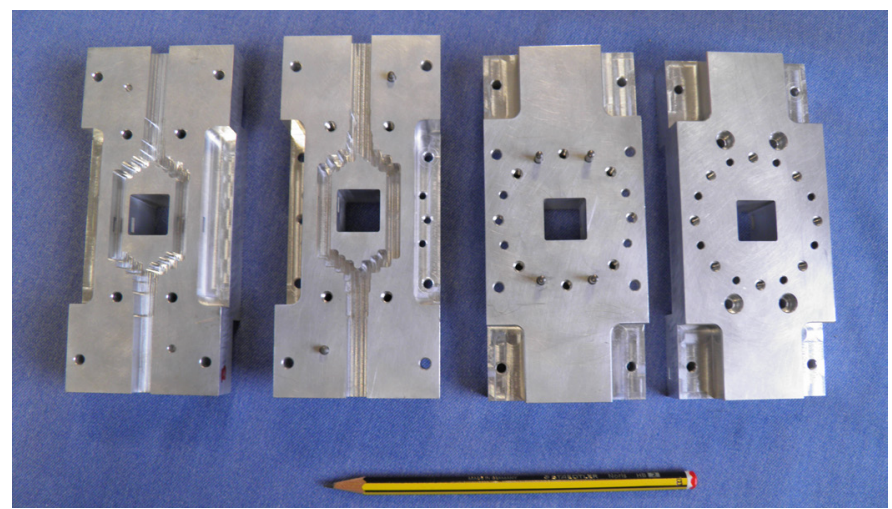

Fig. 5. Four E-plane split-block waveguide parts on aluminum of the double directional coupler to square waveguide prototype. Internal view of one rectangular to square waveguide coupler (two pieces on the left).

\section{EXPERIMENTAL RESULTS}

The S-parameters of the coupler are measured with an Agilent Technologies E8364B vector network analyzer (VNA) using a TRL calibration of the WR62 standard waveguide. The measurements were always two-port, with waveguide terminations in the other rectangular waveguide ports, or a radiating element in the square waveguide with a microwave absorber material placed facing the port. The square waveguide was connected to the VNA through coaxial-to-waveguide WR62 transitions and square-to-circular and circular-torectangular waveguide transitions. All the measurements are compared to electromagnetic simulations done with the Finite Element Method-based software ANSYS ${ }^{\circledR}$ High Frequency Structure Simulation (HFSS) Electronics Desktop, using driven modal solution type and adaptive mesh analysis with convergence set to a maximum delta $\mathrm{S}$ of 0.005 .

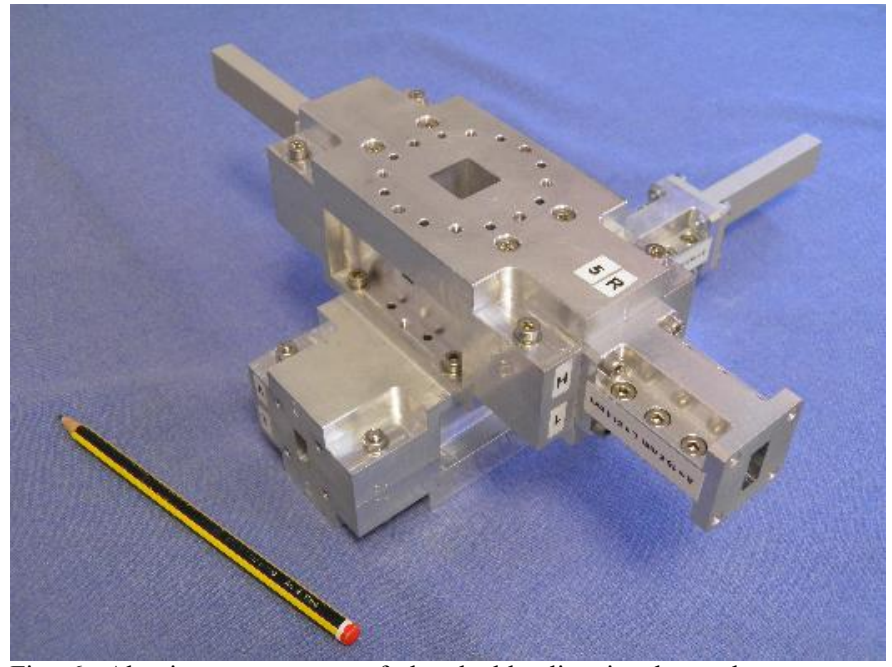

Fig. 6. Aluminum prototype of the double directional coupler to square waveguide with waveguide terminations in two of the rectangular waveguide ports named 3 and 4. Dimensions $193.9 \mathrm{~mm}$ x $193.9 \mathrm{~mm}$ x $106.2 \mathrm{~mm}$.

TABLE I

Dimensions of THE DOUBLE DiRECTIONAL COUPLER to SQUARE WAVEGUIDE

\begin{tabular}{cc|cc} 
Parameter & $\begin{array}{c}\text { Dimension } \\
(\mathrm{mm})\end{array}$ & Parameter & $\begin{array}{c}\text { Dimension } \\
(\mathrm{mm})\end{array}$ \\
\hline \hline a & 15.8 & b & 7.9 \\
a_s & 16.8 & Lh & 6.3 \\
W & 106.2 & Wh & 2.7 \\
W1 & 53.1 & d1 & 67.9 \\
L & 142.5 & d2 & 74.6 \\
L1 & 62.8 & Ls1 & 19.5 \\
Wa & 8.7 & Ls2 & 35.6 \\
Wb & 10.2 & Lr0 & 51.4 \\
Wc & 10.7 & Lr1 & 30.5 \\
Wd & 8.8 & Lr2 & 9.0 \\
We & 11.6 & Lr3 & 4.7 \\
Wf & 8.3 & Lr4 & 2.9 \\
Wg & 8.5 & Lr5 & 7 \\
Wj & 5.8 & Lr6 & 7 \\
\hline \hline
\end{tabular}

The measured and simulated coupling factor for both couplers $(\mathrm{C} 1$ and $\mathrm{C} 2)$ for both propagating modes ( $\mathrm{Y}$ and $\mathrm{X})$ to port\#5 of the square waveguide are depicted in Fig. 7. These results are measured with waveguide terminations in three ports of the rectangular waveguides and a well matched horn facing a microwave absorber material in port\#6. Waveguide terminations are in port\#3 of Coupler\#1, port\#4 of Coupler\#2, and in port\#2 of Coupler\#2 for measuring $\mathrm{C} 1$ or in port\#1 of Coupler\#1 for measuring $\mathrm{C} 2$. The obtained coupling factor to the desired mode is $-31 \pm 3.8 \mathrm{~dB}$ in the frequency band from 10 to $18.9 \mathrm{GHz}$, with $\mathrm{C} 1$ coupling to the mode $\mathrm{V}\left(\mathrm{C}_{5 \mathrm{Y}}\right)$ and $\mathrm{C} 2$ to the mode $\mathrm{H}\left(\mathrm{C}_{5 \mathrm{X}}\right)$. On the other hand, the coupling to the orthogonal modes for both couplers are lower than $-80 \mathrm{~dB}$, except for some frequencies that achieve $-60 \mathrm{~dB}$, measuring with $10 \mathrm{MHz}$ frequency step, which corresponds to an average cross-polarization of $48 \mathrm{~dB}$.

The difference in amplitude and phase of the coupling $\mathrm{C}_{5 \mathrm{Y}}$ and $\mathrm{C}_{5 \mathrm{X}}$ is depicted in Fig. 8. The amplitude and phase errors are $\pm 0.2 \mathrm{~dB}$ and $\pm 1^{\circ}$ from 10 to $18.9 \mathrm{GHz}$.

Subsequently, the isolation measurement for both orthogonal modes is performed from port\#1 or port\#2 to port\#6, with waveguide terminations in three of the rectangular waveguides 
ports and a well matched horn facing a microwave absorber material in port\#5. These isolation measurements together with simulation results are shown in Fig. 9. The obtained isolation is better than $-50 \mathrm{~dB}$ at the low frequencies of the band. The directivity is reduced up to $5 \mathrm{~dB}$ for the high frequencies of the band.

The insertion loss of the square waveguide section itself is lower than $0.3 \mathrm{~dB}$ up to $15 \mathrm{GHz}$ and lower than $0.2 \mathrm{~dB}$ up to $18.9 \mathrm{GHz}$, with return loss better than $15 \mathrm{~dB}$ over the whole frequency band as it is shown in Fig. 10.

Measurements of the coupling and isolation for both waveguide directional couplers to square waveguide showing the symmetry of the double directional coupler are depicted in Fig. 11. For Coupler\#1 both coupling and isolation parameters are for mode $\mathrm{TE}_{01}(\mathrm{Y})$ in port\#5 and for Coupler\#2 both coupling and isolation parameters are for mode $\mathrm{TE}_{10}(\mathrm{X})$ in port\#6.

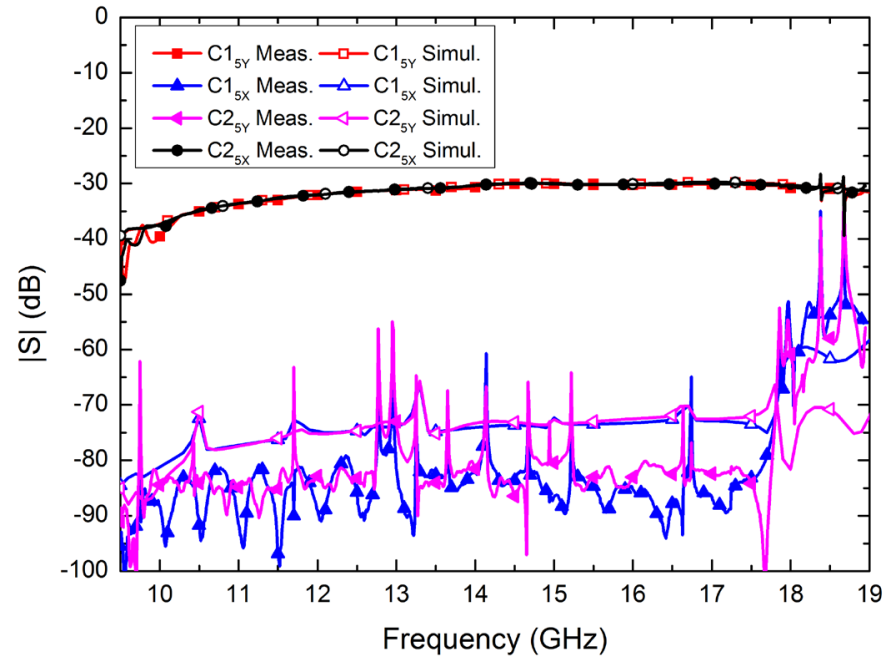

Fig. 7. Simulation and measurements of the coupling for both directional coupler to square waveguide and for both modes $\mathrm{TE}_{01}(\mathrm{Y})$ and $\mathrm{TE}_{10}(\mathrm{X})$. Results with waveguide terminations in port\#3, port\#4, and port\#2 for $\mathrm{C} 1$ or port\#1 for $\mathrm{C} 2$.

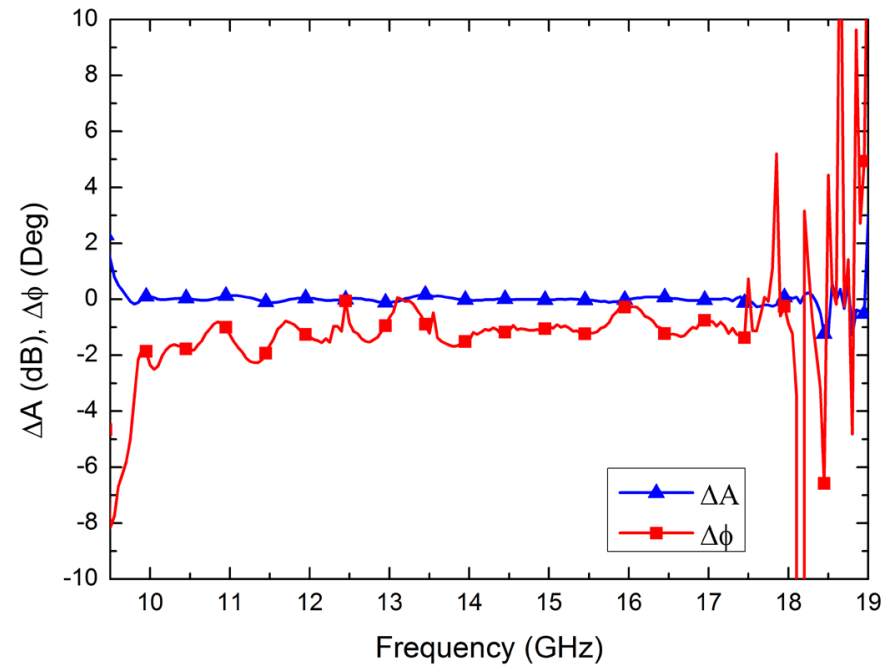

Fig. 8. Simulation and measurements of the amplitude and phase difference between both directional coupler to square waveguide for the coupled modes $\mathrm{TE}_{01}(\mathrm{Y})\left(\mathrm{C}_{5 \mathrm{Y}}\right)$ and $\mathrm{TE}_{10}(\mathrm{X})\left(\mathrm{C} 2_{5 \mathrm{X}}\right)$.

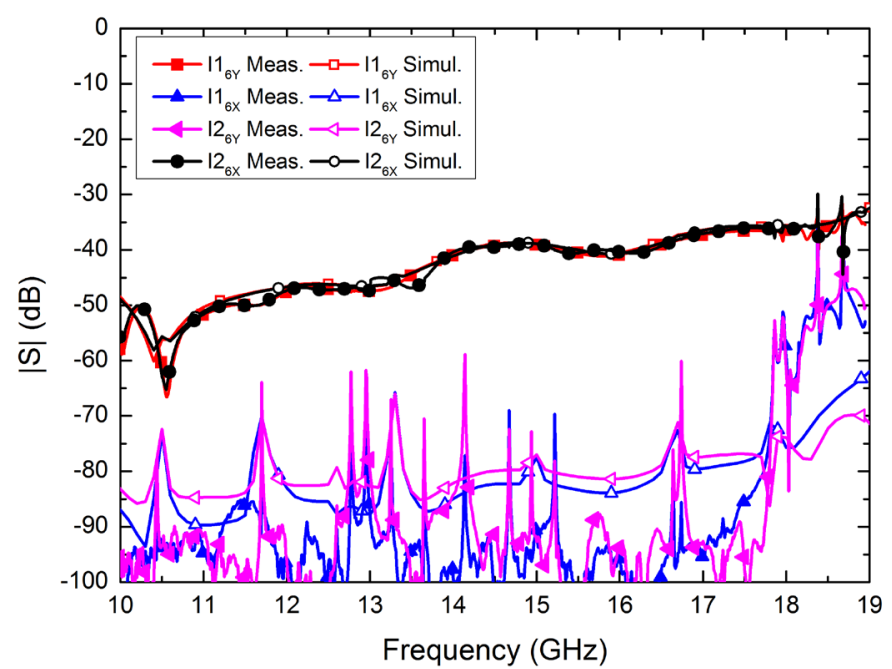

Fig. 9. Simulation and measurements of the isolation for both directional couplers to square waveguide and for both modes $\mathrm{TE}_{01}(\mathrm{Y})$ and $\mathrm{TE}_{10}(\mathrm{X})$. The results are with waveguide terminations in port\#3, port\#4, and port\#2 for I1 or port\#1 for I2.

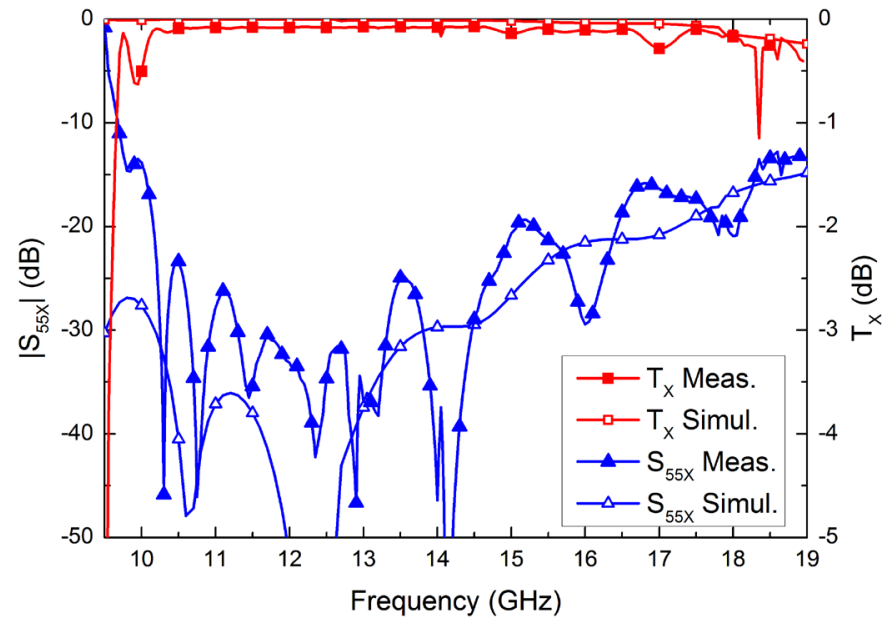

Fig. 10. Simulation and measurements of the transmission and matching for the square waveguide. All the rectangular waveguide ports are with waveguide terminations.

\section{A. Stokes parameters of calibration signals}

The Stokes parameters at the receiver input, relative to two in-phase signals with the same amplitude applied to the coupler, can be calculated using the measured parameters $\mathrm{C} 1_{5 \mathrm{Y}}$ and $\mathrm{C} 2_{5 \mathrm{X}}$ with axis basis $(\hat{x}, \hat{y})$ according to Fig. 2 . The calculated Stokes parameters are normalized respect to the intensity I and they are shown in Fig. 12 (a). The parameter V indicates the level of circularly polarized wave, which appears as a parasitic due to the small phase difference between the two coupled signals depicted in Fig. 8. The component $\sqrt{Q^{2}+U^{2}}$ is the output signal power level with respect to the applied input signal power, and the $\mathrm{V}$ component, which is more than $15 \mathrm{~dB}$ lower, are depicted in Fig. 12 (b). 


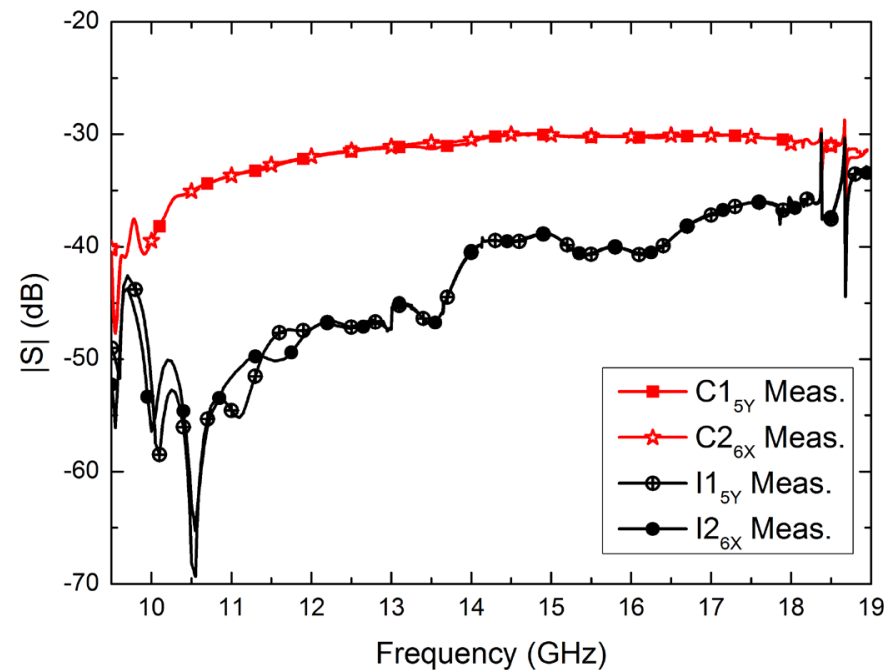

Fig. 11. Measurements of the coupling and isolation for both directional couplers to square waveguide. For Coupler\#1 to mode $\mathrm{TE}_{01}(\mathrm{Y})$ and for Coupler\#2 to mode $\mathrm{TE}_{10}(\mathrm{X})$.

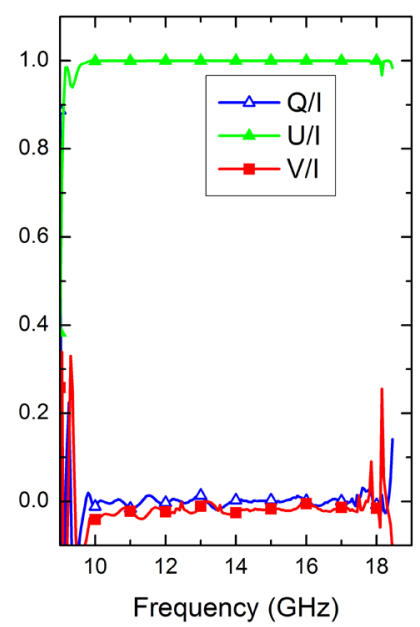

(a)

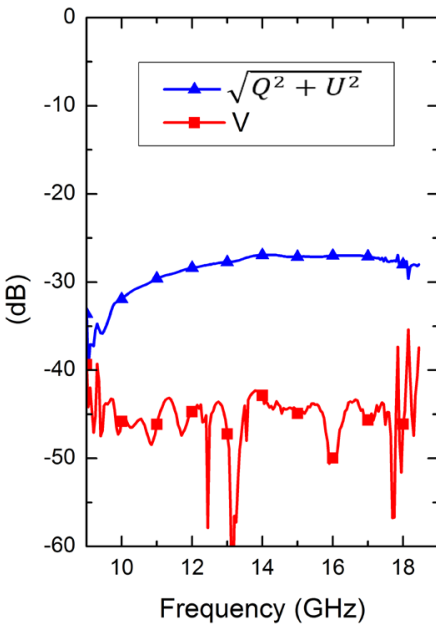

(b)
Fig. 12. (a) Normalized Stokes parameters for two in-phase signals with the same amplitude applied to the coupler calculated with coupling parameters measurement. (b) Measured $\sqrt{Q^{2}+U^{2}}$ and $\mathrm{V}$ components.

The obtained Stokes parameters show that injected in-phase signals and with same amplitude produce a linearly polarized wave of $99.9 \%$ with an angle of polarization of $45^{\circ}$ and below $2 \%$ of circular polarization. A different angle of linear polarization is obtained when the amplitude of the two injected signals is different. Moreover, circular polarization can be obtained with two injected signals with the same amplitude and $90^{\circ}$ out of phase. Finally when both the amplitude and phase of the injected signals are different, an elliptically polarization is obtained. Therefore, the presented coupler allows to generate the three linearly polarized waves and a circular polarized wave needed to calibrate the four parameters of a fully polarimetric radiometer. In the case of the $\mathrm{CMB}$ it is considered that the parameter $\mathrm{V}$ is cero since the $\mathrm{CMB}$ has no circular polarization, then with three linearly polarized waves a polarimetric radiometer intended to measure the $\mathrm{CMB}$ could also be calibrated with the proposed coupler.

\section{CONCLUSION}

A new double waveguide coupler to square waveguide targeted for polarized calibration is presented. This waveguide coupler is designed to couple the two orthogonal modes $\mathrm{TE}_{10}$ and $\mathrm{TE}_{01}$ in a square waveguide through rectangular waveguides, obtaining a known elliptically polarized wave. This subsystem can be placed after the antenna of a polarimetric radiometer, injecting signals that are coupled to the square waveguide to produce at least four brightness temperatures for polarization calibration and measurement of Stokes parameters. The double square waveguide coupler is composed of four Eplane split-block waveguide parts, which are easy to manufacture, and they are assembled in two blocks rotated 90 degrees. Both simulated and measured performances show very good agreement in terms of S-parameters. The measured results demonstrate a full-band double coupler from 10 to $18.9 \mathrm{GHz}$ with average coupling of around $31 \mathrm{~dB}$ and cross polarization better than $50 \mathrm{~dB}$.

\section{REFERENCES}

[1] C. Bischoff, et al., "The Q/U Imaging Experiment Instrument," The Astrophysical Journal, vol. 768, no.1, pp. 768-769, Apr. 2013.

[2] The POLARBEAR Collaboration, et al. "A Measurement of the Cosmic Microwave Background B-mode Polarization Power Spectrum at Subdegree Scales from Two Years of POLARBEAR Data," The Astrophysical Journal, vol. 848, no. 2, pp. 121-136, Oct. 2017.

[3] O.G. King, et al., "The C-Band All-Sky Survey (C-BASS): design and implementation of the northern receiver," Monthly Notices of the Royal Astronomical Society, vol. 438, no. 3, pp. 2426-2439, Mar. 2014,

[4] J.P. Kaufman, et al. "Self-calibration of BICEP1 three-year data and constraints on astrophysical polarization rotation", Physical Rev. D, vol. 89, Mar. 2014.

[5] P. S. Narvekar, G. Heygster, R. Tonboe and T. J. Jackson, "Analysis of WindSat Third and Fourth Stokes Components Over Arctic Sea Ice," IEEE Trans. Geosci. Remote Sensing, vol. 49, no. 5, pp. 1627-1636, May 2011.

[6] A. Duric, A. Magun, A. Murk, C. Matzler and N. Kampfer, "The Fully Polarimetric Imaging Radiometer SPIRA at $91 \mathrm{GHz}$," IEEE Trans. Geosci. Remote Sensing, vol. 46, no. 8, pp. 2323-2336, Aug. 2008.

[7] O. Stahli, C. Mätzler, A. Murk and N. Kämpfer, "Sky measurements with the imaging polarimeter SPIRA at $91 \mathrm{GHz}, "$ in Proc. 11th Specialist Meeting on Microw. Radiometry and Remote Sensing of the Environment, Washington, DC, Mar. 2010, pp. 181-186.

[8] J. R. Piepmeier, L. Hong and F. A. Pellerano, "Aquarius L-Band Microwave Radiometer: 3 Years of Radiometric Performance and Systematic Effects," IEEE J. Sel. Top. Appl. Earth Observations Remote Sensing, vol. 8, no. 12, pp. 5416-5423, Dec. 2015.

[9] J. R. Piepmeier et al., "SMAP L-Band Microwave Radiometer: Instrument Design and First Year on Orbit," IEEE Trans. Geosci. Remote Sensing, vol. 55, no. 4, pp. 1954-1966, Apr. 2017.

[10] N. Skou, B. Laursen and S. Sobjaerg, "Polarimetric radiometer configurations: potential accuracy and sensitivity," IEEE Trans. Geosci. Remote Sens., vol. 37, no. 5, pp. 2165-2171, Sep. 1999.

[11] R.H. Dicke, "The measurement of thermal radiation at microwave frequencies," Rev. Sci. Instr., vol. 17, pp. 268-275, Jul. 1946.

[12] I. Corbella, A. J. Gasiewski, M. Klein, V. Leuski, A. J. Francavilla and J. R. Piepmeier, "On-board accurate calibration of dual-channel radiometers using internal and external references," IEEE Trans. Microw. Theory Tech., vol. 50, no. 7, pp. 1816-1820, Jul. 2002.

[13] P. A. R. Ade, et al. "A Measurement of the Cosmic Microwave Background B-mode Polarization Power Spectrum at Sub-degree with POLARBEAR," The Astrophysical Journal, vol. 794, no. 2, pp.171-192, Oct. 2014.

[14] A. J. Gasiewski and D. B. Kunkee, "Calibration and applications of polarization-correlating radiometers," IEEE Trans. Microw. Theory Tech., vol. 41, no. 5, pp. 767-773, May 1993. 
[15] C. W. O'Dell, D. S. Swetz and P. T. Timbie, "Calibration of millimeterwave polarimeters using a thin dielectric sheet" IEEE Trans. Microw. Theory Tech., vol. 50, no. 9, pp. 2135-2141, Sep. 2002.

[16] Y. D. Takahashi, et al. "Characterization of the BICEP Telescope for HighPrecision Cosmic Microwave Background Polarimetry," The Astrophysical Journal, vol. 711, no. 2, pp. 1141-1156, Mar. 2010.

[17] O. Tajima, H. Nguyen, C. Bischoff, A. Brizius, I. Buder, and A. Kusaka, "Novel Calibration System with Sparse Wires for CMB Polarization Receivers," J Low Temp Phys., vol. 167, no. 5-6, pp. 936-942, Jun. 2012.

[18] J. Lahtinen, A. J. Gasiewski, M. Klein and I. S. Corbella, "A calibration method for fully polarimetric microwave radiometers," IEEE Trans. Geosci. Remote Sens., vol. 41, no. 3, pp. 588-602, Mar. 2003.

[19] J. Lahtinen and M. T. Hallikainen, "HUT fully polarimetric calibration standard for microwave radiometry," IEEE Trans. Geosci. Remote Sens., vol. 41, no. 3, pp. 603-611, Mar. 2003.

[20] N. Ou et al., "A novel method for constructing the end-to-end calibration matrix of fully polarimetric radiometer," in Int. Conf. on Microw. and Millimeter Wave Techn. (ICMMT), Shenzhen, 2012, pp. 1-4.

[21] J. Lahtinen and M. T. Hallikainen, "Retardation Plate for the Calibration of a Fully Polarimetric Radiometer: Determination of Characteristics," IEEE Trans. Geosci. Remote Sens., vol. 10, no. 7, pp. 3046-3054, Jul. 2017.

[22] Peng and C. S. Ruf, "Calibration Method for Fully Polarimetric Microwave Radiometers Using the Correlated Noise Calibration Standard," IEEE Trans. Geosci. Remote Sensing, vol. 46, no. 10, pp. 3087-3097, Oct. 2008.

[23] O. A. Peverini et al., "On-board calibration system for millimeter-wave radiometers based on reference polarized signal injection," IEEE Trans. Microw. Theory Tech., vol. 54, no. 1, pp. 412-420, Jan. 2006,

[24] G. G. Gentili, L. Lucci, R. Nesti, G. Pelosi and S. Selleri, "A Novel Design for a Circular Waveguide Directional Coupler," IEEE Trans. Microw. Theory Tech., vol. 57, no. 7, pp.1840-1849, Jul. 2009.

[25] T. Yuan, X. Yin, H. Zhao, J. Shi, W. Zhong and Q. Liu, "A design of Qband noise injector," in Proc. of 2014 3rd Asia-Pacific Conf. on Antennas and Propagation, Harbin, Jul. 2014, pp. 1162-1164.

[26] H. A. Bethe, "Theory of Diffraction by Small Holes," Phys. Rev., vol. 66, no. 7-8, pp. 163-182, Oct. 1944.

[27] S. B. Cohn and R. Levy, "History of Microwave Passive Components with Particular Attention to Directional Couplers," IEEE Trans. Microw. Theory Tech., vol. 32, no. 9, pp. 1046-1054, Sep. 1984.

[28] H. J. Riblet and T. S. Saad, "A New Type of Waveguide Directional Coupler," in Proc. IRE, vol. 36, no. 1, pp. 61-64, Jan. 1948.

[29] R. Levy, "Analysis and Synthesis of Waveguide Multi-Aperture Directional Couplers," in Proc. G-MTT Int. Microw. Symp., Detroit, MI, USA, 1968, pp. 32-38.

[30] R. Levy, "Improved single and multiaperture waveguide coupling theory, including explanation of mutual interactions," IEEE Trans. Microw. Theory Tech., vol. 28, no. 4, pp. 331-338, Apr. 1980.

[31] Y. Zhang, Q. Wang and J. Ding, "A Cross-Guide Waveguide Directional Coupler with High Directivity and Broad Bandwidth," IEEE Microw. Wireless Compon. Lett., vol. 23, no. 11, pp. 581-583, Nov. 2013.

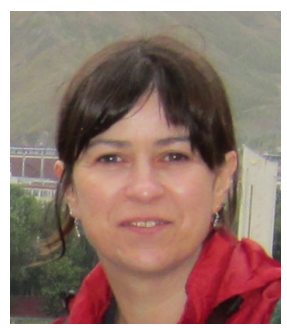

Beatriz Aja received the Telecommunications Engineering degree and the Ph.D. in 1999 and 2007 respectively from the University of Cantabria, Spain.

Since 2014 she is Assistant Professor in the Department of Communications Engineering at the University of Cantabria, Spain.

From 2008 to 2012 and from 2013 to 2015 she was invited scientist at the Fraunhofer Institute for Applied Solid State Physics (Germany), in a joint collaboration project with Centro Astronómico de Yebes (Spain), where her main activity was the design of Monolithic Microwave Integrated Circuits (MMIC) for cryogenic applications. She has been involved in development of the receivers for the Planck Mission and QUIJOTE (Q-U-I JOint Tenerife) CMB Experiment. Her research interests include design and characterization of LNAs for cryogenic applications, microwave and millimeter-wave passive components and radio astronomy receivers.

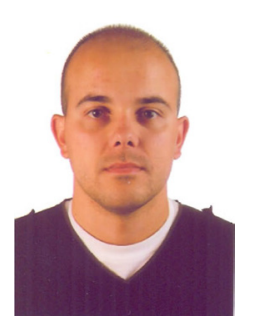

Enrique Villa Enrique Villa was born in Santander, Spain. He received the Telecommunications Engineering, Master's degree in Information Technologies and Mobile Networks Communications and Ph.D. degrees from the University of Cantabria, Santander, Spain, in 2005, 2008 and 2014.

From 2006 to 2017, he was a researcher with the University of Cantabria, where he participated in the design of low-noise, high-sensitivity, and broadband microwave receivers for radio astronomy. Since 2018, he is a researcher with the Instituto de Astrofisica de Canarias (IACTec Department), where he currently develops microwave radiometer systems to be applied in biomedical applications, which measure natural radiation from biological tissues within the human body

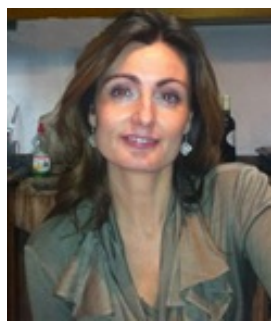

Luisa de la Fuente graduated from the University of Cantabria, Santander, Spain in 1991 and received the doctoral degree in Electronics Engineering in 1997. From 1992 to 1993, she was an Associate Teacher in the Department of Electronics, University of Cantabria. She is currently a lecturer in the Department of Communication Engineering, University of Cantabria. Her main research interests include design and testing of microwave circuits in both hybrid and monolithic technologies. In the last years she has worked in projects focussed in the development of radiometers for space applications, like the Planck Mission, in particular in low noise amplifiers at room and cryogenic temperatures. Currently she is involved in several projects focused on the development of very low noise receivers in the 30 and $40 \mathrm{GHz}$ frequency bands for the QUIJOTE experiment, and on the development of polarimetry receivers at $\mathrm{W}$ band.

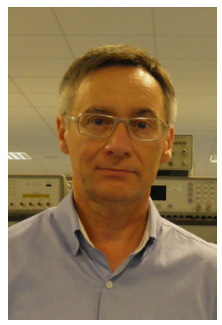

Eduardo Artal (M'81) received the Engineer and Dr. Engineer in Telecommunication degrees from the Technical University of Catalonia, Barcelona, Spain, in 1976 and 1982, respectively. From 1976 to 1990 he was an Assistant Professor with the Technical University of Catalonia. From 1979 to 1981, in a partial leave from the university, he joined Mier Allende S.A., Barcelona, Spain, where he was involved with TV and FM radio re-emitters development. Since 1990 he has been a Professor at the University of Cantabria, Santander, Spain, where he was manager of the Telecommunication Engineering course from 1990 to 1994. From 1994 to 1998 he was manager of the National Program for Information and Communications Technologies at the "Plan Nacional de I+D", National R\&D Plan in the Spanish Ministry of Education and Science, Madrid. His main areas of activities and contributions have been: microwave circuits and systems, including monolithic microwave integrated circuits up to $50 \mathrm{GHz}$. His current research interests are low noise millimetre-wave amplifiers and receivers for radio-astronomy applications 